\title{
Mining Android Apps for Anomalies
}

\author{
Konstantin Kuznetsov ${ }^{\mathrm{a}}$, Alessandra Gorla ${ }^{\mathrm{b}}$, Ilaria Tavecchia ${ }^{\mathrm{c}}$, Florian Gross ${ }^{\mathrm{a}}$, \\ Andreas Zeller ${ }^{\mathrm{a}}$ \\ ${ }^{a}$ Saarland University, Saarbrücken, Germany \\ ${ }^{b}$ IMDEA Software Institute, Madrid, Spain \\ ${ }^{c}$ SWIFT, Bruxelles, Belgium
}

\begin{abstract}
How do we know a program does what it claims to do? Our CHABADA prototype can cluster Android apps by their description topics and identify outliers in each cluster with respect to their API usage. A "weather" app that sends messages thus becomes an anomaly; likewise, a "messaging" app would typically not be expected to access the current location. In this paper we present a new approach for anomaly detection that improves the classification results of our original CHABADA paper [1]. Applied on a set of 22,500+ Android applications, our CHABADA prototype can now predict $74 \%$ of novel malware as such, without requiring any known malware patterns, maintaining the false positive rate close to $10 \%$.

Keywords: Android apps, malware detection, application behavior, app mining, description analysis
\end{abstract}

\section{Introduction}

Detecting whether a mobile application behaves as expected is a prominent problem for users. Whenever they install a new app on their mobile device, they run the risk of it being "malware" - that is, to act against their interests. Security researchers have largely focused on detecting malware in Android apps, but their techniques typically check new apps against a set of predefined known patterns of malicious behavior. This

Email addresses: kuznetsovecs. uni-saarland. de (Konstantin Kuznetsov), alessandra.gorla@imdea . org (Alessandra Gorla), fgross@cs. uni-saarland. de (Florian Gross), zellerecs. uni-saarland. de (Andreas Zeller) 


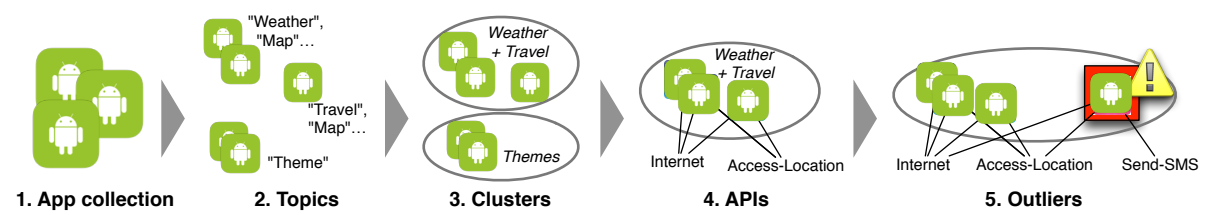

Figure 1: Detecting applications with anomalous behavior. Starting from a collection of "benign" apps (1), CHABADA identifies their description topics (2) to form clusters of related apps (3). For each cluster, CHABADA identifies the APIs used (4), and can then identify outliers that use APIs that are uncommon for that cluster (5).

approach works well to detect new malware that uses known patterns, but does not help against new attack patterns. Moreover, in Android it is not easy to define what malicious behavior is, and therefore to define what are the key features to detect malware. The problem is that any specification on what makes behavior beneficial or malicious very much depends on the current context.

Typical Android malware, for instance, sends text messages to premium numbers, or collects sensitive information of the user, such as the mobile number, the current location, and the list of emails associated to different accounts. However, this very same information, and the very same operations, frequently occur in benign applications as well. Sending text messages to premium numbers is for instance a legitimate paying method to unlock new app features; Tracking the current location is what a navigation app has to do; Collecting the list of contacts and sending it to an external server is what most free messaging apps like WhatsApp do upon synchronization. The question thus is not whether the behavior of an app matches a specific malicious pattern or not; it is whether an app behaves as one would expect.

In our previous work we presented CHABADA, a technique to check implemented app behavior against advertised app behavior [1]. We analyzed the natural language descriptions of 22.500+ Android applications, and we checked whether the description matched the implemented behavior, represented as a set of application programming interfaces (APIs). The key of CHABADA is to associate descriptions and API usage to detect anomalies.

Our CHABADA approach runs over the five steps illustrated in Figure 1. 
1. CHABADA starts with a collection of 22,500+ supposedly "benign" Android applications downloaded from the Google Play Store.

2. Using Latent Dirichlet Allocation (LDA) on the app descriptions, CHABADA identifies the main topics ("theme", "map", "weather", "download") for each application.

3. CHABADA then clusters applications by related topics. For instance, if there were enough apps whose main description topics are "navigation" and "travel", they would form one cluster.

4. In each cluster, CHABADA identifies the APIs each app statically accesses. It only considers sensitive APIs, which are governed by a user permission. For instance, APIs related to Internet access are controlled by the "INTERNET" permission.

5. Using unsupervised learning, CHABADA identifies outliers within a cluster with respect to API usage. It produces a ranked list of applications for each cluster, where the top apps are most abnormal with respect to their API usage—indicating possible mismatches between description and implementation. Unknown applications would thus first be assigned to the cluster implied by their description, and then be classified as being normal or abnormal.

By flagging anomalous API usage within each cluster, CHABADA is set up to detect any suspicious app within a set of similar apps, and can therefore detect whether an app has any mismatch between advertised and implemented behavior. We show how this works in practice with a real app as an example. Figure 2 shows the description of the Official New York Jets team app available from the Google Play Store. Its description clearly puts it into the "sports" cluster.

Beside expected common API calls, the instance of the Official New York Jets app that we analyzed can check whether GPS location is available, via the API method LocationManager.addGpsStatusListener(), and can send text messages, via the API method SmsManager.sendTextMessage(), which are highly uncommon operations for

[https://play.google.com/store/apps/details?id=com.yinzcam.nfl.jets 
This is the official mobile app of the New York Jets. Make your Android device a unique part of your game-day experience for Jets games. Want to catch breaking news of the team? See real-time statistics for every drive? Watch video-on-demand clips of press conferences and player interviews? Follow postgame blogs and pre-game previews of the matchups? Now, you can stay in touch with the Jets anytime, anywhere, on your Android device.

FEATURES:

- News: Real-time breaking news from the Jets, previews of upcoming matchups, post-game blogs

- Video: Video-on-demand clips of Jets' press conferences, coach and player interviews

- Photos: Gallery of game-time action

- Audio: Podcasts

- Stats: Real-time statistics and scores from the official NFL stats engine, head-to-head stats of the matchup, player stats, drive-by-drive stats, box score, out-of-town scores around the league

- Standings: Division and conference standings

- Fantasy: Keep track of your favorite fantasy players

- Depth chart: Shown by offense, defense and special teams

- Social media: Aggregated Twitter of all of your favorite Jets tweeps, check in to the stadium on game-day, one-click tweet of all media items, one-click Facebook posting of all media items

- Digital keepsake: Your game-time moment superimposed on the stadium jumbotron in the form of a unique digital keepsake

- Schedule: Schedule of upcoming games, and scores/stats of previous games from the season, ticket purchase for games

- Concessions: Interactive map of MetLife Stadium with searchable concessions-stands and amenities

- Problem-reporting: Reports of various problems and issues around the stadium

- Evolving home-screen: Pre-game, in-game, post-game, off-season countdown, draft-day

- In-stadium live video and replays: Limited beta test; connect to the MLSOpen Wi-Fi network at MetLife Stadium

Follow us @ nyjets on Twitter for updates or visit www.newyorkjets.com. SUPPORT/QUESTIONS; Email support@yinzcam.com or send a tweet to @yinzcam The Official New York Jets app is created and maintained by YinzCam, Inc., on behalf of the New York Jets.

Figure 2: Official New York Jets app description

this kind of applications. These API method calls, together with similar others, make Official New York Jets an outlier within the "sports" cluster. By flagging such anomalies, CHABADA can detect false advertising, plain fraud, masquerading, and other questionable behavior. CHABADA can be used as a malware detector as well. By training it on a sample of benign apps, CHABADA can classify new apps as benign or malware, without any previous notion of malicious behavior.

This chapter extends our previous conference paper [1] by presenting several new techniques that lead to significant improvements: 
1. We now rank down irrelevant APIs when looking for anomalies. Specifically, we give a lower weight to APIs that are common within a particular cluster (e.g., Internet access, which is frequently used in applications). By giving more importance to less common behavior, CHABADA can highlight outliers more easily.

2. We incorporate an additional technique for anomaly detection. The anomaly detection of CHABADA is now based on a distance-based algorithm, which allows to clearly identify the APIs that make an app anomalous.

3. To use CHABADA as a classifier of malicious applications, we now run anomaly detection as a preliminary step, and we exclude the anomalies from the training set. This allows to remove noise from the training set, and consequently to improve the abilities of the classifier. CHABADA can now predict $74 \%$ of malware as such (previously $56 \%$ ), and suffers from only $11 \%$ of false positives (previously $15 \%$ ).

4. We can now automatically select the optimal parameters for the classifier. This also contributes to the improvement of CHABADA as a malware classifier.

The remainder of this paper is organized as follows. We first describe how CHABADA clusters applications by description topics in Section 2. This book chapter does not improve our first paper [1] on this side, but we include a description of this step for the sake of completion. Section 3 describes how in each cluster we detect outliers with respect to their API usage. In particular we describe the new algorithm that CHABADA uses, and we highlight the advantages of the new approach. Section 4 evaluates the improvements of CHABADA. After discussing the related work Section 5, Section 6 closes with conclusions and future work.

\section{Clustering Apps by Description}

The intuition behind CHABADA is simple: applications that are similar, in terms of their descriptions, should also behave similarly. As a consequence, applications that behave differently from their similars should be further inspected, as they may 
have malicious behavior. For this, we must first establish what makes two descriptions "similar". We start with describing our Android apps collection, and how we collected it (Section 2.1). After initial processing (Section 2.2), CHABADA identifies topics of app descriptions (Section 2.3), and then clusters the apps based on common topics Section 2.4 to Section 2.6.

\subsection{Collecting Applications}

CHABADA is based on detecting anomalies from "normal", hopefully benign applications. As a base for such "normal" behavior, CHABADA relies on a large set of applications from the Google Play Store, the central resource for Android apps. Our automated crawler ran at regular intervals (i.e. every two weeks) during the Winter and Spring of 2013, and for each of the 30 categories in the Google Play Store, downloaded the top 150 free ${ }^{2}$ applications in each category. A single complete run of our script thus returned 4,500 apps; as the top 150 apps shifted during our collection, we obtained a total of 32,136 apps across all categories.

In addition to the actual app (coming as an APK file), we also collected the store metadata—such as name and description.

\subsection{Preprocessing Descriptions with NLP}

Before subjecting app descriptions to topic analysis, CHABADA applies standard techniques of natural language processing (NLP) for filtering and stemming [2].

App descriptions in the Google Play Store frequently contain paragraphs in multiple languages-for instance, the main description is in English, while at the end of the description developers add a short sentence in different languages to briefly describe the application. To be able to cluster similar descriptions, CHABADA has to work on a single language, and because of its predominance we chose English. CHABADA relies

on Google's Compact Language Detector ${ }^{3}$ to detect the most likely language of the app description, and it removes non-English paragraphs.

2 Section 4.4 discusses possible induced bias.

http://code.google.com/p/chromium-compact-language-detector 
After multi-language filtering, CHABADA removes stop words (common words such as "the", "is", "at", "which”, "on”, .. ), and applies stemming on all descriptions employing the power of Natural Language Toolki $\rrbracket^{4}$ Stemming is a common NLP technique to identify the word's root, and it is essential to make words such as "playing", "player", and "play" all match to the single common root "plai". Stemming can improve the results of later NLP processes, since it reduces the number of words. CHABADA also removes non-text items such as numerals, HTML tags, links and email addresses thanks to specific Python modules such as HTMLParser 5

As an example, consider the description of Official New York Jets in Figure 2, after the preprocessing phase, it appears as:

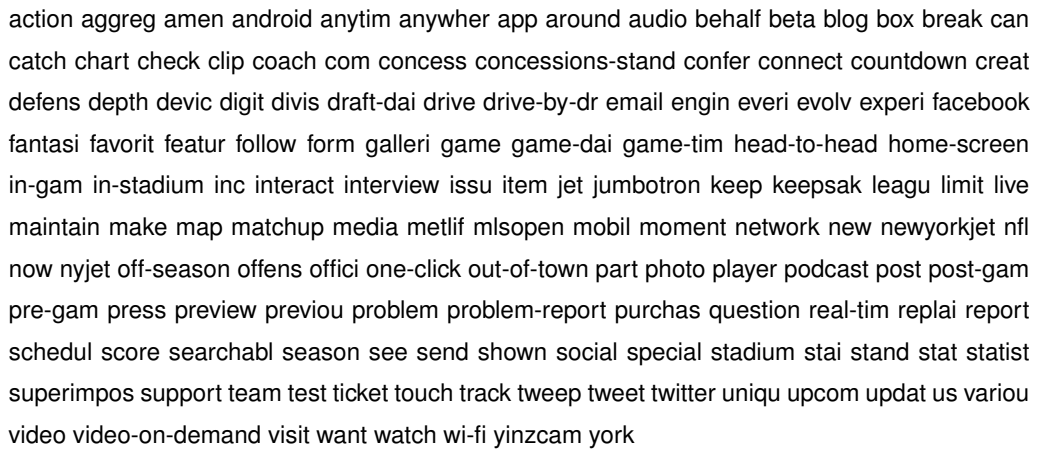

We remove from our data set those applications whose description has less than 10 words after the preprocessing we just described. Also, we eliminate all applications without any sensitive APIs (see Section 3 for details). This resulted in a final set of 22,521 apps, which form the base for CHABADA.

\subsection{Identifying Topics with LDA}

To identify sets of topics for the apps under analysis, CHABADA resorts to topic modeling using Latent Dirichlet Allocation (LDA) [3].

LDA is an unsupervised, statistical algorithm that discovers latent semantic topics in a collection of text documents. LDA represents documents as random mixtures over

${ }^{4}$ http://www.nltk.org

5 https://docs.python.org/2/library/htmlparser.html 
multiple latent topics, where each "topic" is characterized by a distribution over a fixed vocabulary of words. Given a set of documents and the number of topics, LDA produces the probability distribution of each topic-document pair and of each word-topic pair, and consequently learns, for each topic, a set of words.

By analyzing a set of app descriptions on sports and social networking, for instance, LDA would group words such as "team", "soccer", "league", and "sport" into one topic, and "share", "facebook", "twitter", and "suggest" into another topic. Applications whose description is mainly about sports would thus be assigned to the first topic, since most of the words occurring in the description belong to the first group. Applications such as the Official New York Jets, however, would be assigned to both topics, as the words in the description appear in both groups.

CHABADA feeds the NLP pre-processing output (i.e., the English text without stop words, and after stemming) into the Mallet framework [4]. CHABADA can be freely configured to choose the number of topics to be identified by LDA; by default it identifies 30, the number of categories covered by the apps in the Google Play Store. Furthermore, by default CHABADA is configured such that an app can belong to at most 4 topics. Limiting the number of topics an app can belong to makes the clustering more effective in our experience.

Table 1 shows the resulting list of topics for the 22,521 descriptions that we analyzed with CHABADA; the "assigned name" is the abstract concept we assigned to that topic. Our example application, Official New York Jets, is assigned to these four topics:

- Topic 24 (“sports") with a probability of $63.1 \%$,

- Topic 8 ("share") with a probability of $17.7 \%$,

- Topic 10 (“files and videos") with a probability of $10.4 \%$, and

- Topic 6 ("game”) with a probability of $6.7 \%$.

\subsection{Clustering Apps with $K$-means}

Topic modeling can assign application descriptions to topics with a certain probability. What we want, however, is to cluster applications with similar descriptions within 
Table 1: Topics mined from Android Apps

\begin{tabular}{|c|c|c|}
\hline Id & Assigned Name & Most Representative Words (stemmed) \\
\hline 0 & "personalize" & galaxi, nexu, device, screen, effect, instal, customis \\
\hline 1 & "game and cheat sheets" & game, video, page, cheat, link, tip, trick \\
\hline 2 & "money" & slot, machine, money, poker, currenc, market, trade, stock, casino coin, finance \\
\hline 3 & "tv" & tv, channel, countri, live, watch, germani, nation, bbc, newspap \\
\hline 4 & "music" & music, song, radio, play, player, listen \\
\hline 5 & "holidays" and religion & christmas, halloween, santa, year, holiday, islam, god \\
\hline 6 & "navigation and travel" & map, inform, track, gps, navig, travel \\
\hline 7 & "language" & language, word, english, learn, german, translat \\
\hline 8 & "share" & email, ad, support, facebook, share, twitter, rate, suggest \\
\hline 9 & "weather and stars" & weather, forecast, locate, temperatur, map, city, light \\
\hline 10 & "files and video" & file, download, video, media, support, manage, share, view, search \\
\hline 11 & "photo and social" & photo, friend, facebook, share, love, twitter, pictur, chat, messag, galleri, hot, send social \\
\hline 12 & "cars" & car, race, speed, drive, vehicl, bike, track \\
\hline 13 & "design and art" & life, peopl, natur, form, feel, learn, art, design, uniqu, effect, modern \\
\hline 14 & "food and recipes" & recip, cake, chicken, cook, food \\
\hline 15 & "personalize" & theme, launcher, download, install, icon, menu \\
\hline 16 & "health" & weight, bodi, exercise, diet, workout, medic \\
\hline 17 & "travel" & citi, guid, map, travel, flag, countri, attract \\
\hline 18 & "kids and bodies" & kid, anim, color, girl, babi, pictur, fun, draw, design, learn \\
\hline 19 & "ringtones and sound" & sound, rington, alarm, notif, music \\
\hline 20 & "game" & game, plai, graphic, fun, jump, level, ball, 3d, score \\
\hline 21 & "search and browse" & search, icon, delet, bookmark, link, homepag, shortcut, browser \\
\hline 22 & "battle games" & story, game, monster, zombi, war, battle \\
\hline 23 & "settings and utils" & screen, set, widget, phone, batteri \\
\hline 24 & "sports" & team, football, leagu, player, sport, basketbal \\
\hline 25 & "wallpapers" & wallpap, live, home, screen, background, menu \\
\hline 26 & "connection" & device, connect, network, wifi, blootooth, internet, remot, server \\
\hline 27 & "policies and ads" & live, ad, home, applovin, notif, data, polici, privacy, share, airpush, advertis \\
\hline 28 & "popular media" & seri, video, film, album, movi, music, award, star, fan, show, gangnam, top, bieber \\
\hline 29 & "puzzle and card games" & game, plai, level, puzzl, player, score, challeng, card \\
\hline
\end{tabular}

the same group. It would appear reasonable to consider obtained topics as separate clusters, but unfortunately topic modeling does not provide a binary decision of whether a description belongs to a particular topic. Moreover, each description may be related to many topics, and even with equal probability, so it would not be clear how to pick a specific cluster for a given application description.

As a consequence, CHABADA uses $K$-means [5], one of the most common clustering algorithms, to group applications with similar descriptions, and it does so by using topic probabilities as features. Given a set of elements and the number of clusters $K$ to be identified, K-means selects one centroid for each cluster, and then associates each element of the data set to the nearest centroid, thus identifying clusters. It should be noted that using words instead of topics would significantly increase the dimension of the feature space, and would thus make $K$-means almost ineffective. 
In this context, we use applications as the elements to be clustered, and we use the probabilities of belonging to topics as features. As an example, Table 2 shows four applications $\mathrm{app}_{1}$ to $\mathrm{app}_{4}$, with the corresponding probabilities of belonging to topics. If we applied K-means to partition the set of applications into two clusters, it would create one cluster with $a p p_{1}$ and $a p p_{3}$, and a second cluster with $a p p_{2}$ and $a p p_{4}$.

Table 2: Four applications and their likelihoods of belonging to specific topics

\begin{tabular}{rrrrr} 
Application & topic $_{1}$ & topic $_{2}$ & topic $_{3}$ & topic $_{4}$ \\
\hline app $_{1}$ & 0.60 & 0.40 & - & - \\
app $_{2}$ & - & - & 0.70 & 0.30 \\
app $_{3}$ & 0.50 & 0.30 & - & 0.20 \\
app $_{4}$ & - & - & 0.40 & 0.60
\end{tabular}

\subsection{Finding the Best Number of Clusters}

One of the challenges with K-means is to estimate the number of clusters that should be created. The algorithm needs to be given either some initial potential centroids, or the number $K$ of clusters to identify. There exist several approaches to identify the best solution, among a set of possible clustering solutions. Therefore, CHABADA runs $\mathrm{K}$-means several times, each time with a different $K$ number, to obtain a set of clustering solutions it would then be able to evaluate. The range for $K$ covers solutions among two extremes: having a small number of clusters (even just 2) with a large variety of apps; or having many clusters (potentially even one per app) and thus being very specific. CHABADA fixes num_topics $\times 4$ as an upper bound, since according to the default settings an application can belong to up to 4 topics.

To identify the best solution, i.e., the best number of clusters, CHABADA uses the elements silhouette, as discussed in [6]. The silhouette of an element is the measure of how closely the element is matched to the other elements within its cluster, and how loosely it is matched to other elements of the neighboring clusters. When the value of the silhouette of an element is close to 1 , it means that the element is in the appropriate cluster. If the value is close to -1 , instead, it means that the element is in the wrong cluster. Thus, to identify the best solution, CHABADA computes the average of the 
Table 3: Clusters of applications. "Size" is the number of applications in the respective cluster. "Most Important Topics" list the three most prevalent topics; most important (> 10\%) shown in bold. Topics less than $1 \%$ not listed.

\begin{tabular}{|c|c|c|c|}
\hline Id & Assigned Name & Size & Most Important Topics \\
\hline 1 & "sharing" & 1,453 & share $(53 \%)$, settings and utils, navigation and travel \\
\hline 2 & "puzzle and card games" & 953 & puzzle and card games $(78 \%)$, share, game \\
\hline 3 & "memory puzzles" & 1,069 & puzzle and card games $(40 \%)$, game $(12 \%)$, share \\
\hline 4 & "music" & 714 & music $(58 \%)$, share, settings and utils \\
\hline 5 & "music videos" & 773 & popular media $(44 \%)$, holidays and religion $(20 \%)$, share \\
\hline 6 & "religious wallpapers" & 367 & holidays and religion $(56 \%)$, design and art, wallpapers \\
\hline 7 & "language" & 602 & language $(67 \%)$, share, settings and utils \\
\hline 8 & "cheat sheets" & 785 & game and cheat sheets $(76 \%)$, share, popular media \\
\hline 9 & "utils" & 1,300 & settings and utils $(62 \%)$, share, connection \\
\hline 10 & "sports game" & 1,306 & game $(63 \%)$, battle games, puzzle and card games \\
\hline 11 & "battle games" & 953 & battle games $(60 \%)$, game $(11 \%)$, design and art \\
\hline 12 & "navigation and travel" & 1,273 & navigation and travel $(64 \%)$, share, travel \\
\hline 13 & "money" & 589 & money $(57 \%)$, puzzle and card games, settings and utils \\
\hline 14 & "kids" & 1,001 & kids and bodies $(62 \%)$, share, puzzle and card games \\
\hline 15 & "personalize" & 304 & personalize $(71 \%)$, wallpapers $(15 \%)$, settings and utils \\
\hline 16 & "connection" & 823 & connection $(63 \%)$, settings and utils, share \\
\hline 17 & "health" & 669 & health $(63 \%)$, design and art, share \\
\hline 18 & "weather" & 282 & $\begin{array}{l}\text { weather and stars }(61 \%) \text {, settings and utils }(11 \%) \text {, navigation and } \\
\text { travel }\end{array}$ \\
\hline 19 & "sports" & 580 & sports $(62 \%)$, share, popular media \\
\hline 20 & "files and videos" & 679 & files and videos $(63 \%)$, share, settings and utils \\
\hline 21 & "search and browse" & 363 & search and browse $(64 \%)$, game, puzzle and card games \\
\hline 22 & "advertisements" & 380 & policies and ads $(97 \%)$ \\
\hline 23 & "design and art" & 978 & design and art $(48 \%)$, share, game \\
\hline 24 & "car games" & 449 & cars $(51 \%)$, game, puzzle and card games \\
\hline 25 & "tv live" & 500 & tv $(57 \%)$, share, navigation and travel \\
\hline 26 & "adult photo" & 828 & photo and social $(59 \%)$, share, settings and utils \\
\hline 27 & "adult wallpapers" & 543 & wallpapers $(51 \%)$, share, kids and bodies \\
\hline 28 & "ad wallpapers" & 180 & policies and ads $(46 \%)$, wallpapers, settings and utils \\
\hline 29 & "ringtones and sound" & 662 & ringtones and sound $(68 \%)$, share, settings and utils \\
\hline 30 & "theme wallpapers" & 593 & wallpapers $(90 \%)$, holidays and religion, share \\
\hline 31 & "personalize" & 402 & personalize $(86 \%)$, share, settings and utils \\
\hline 32 & "settings and wallpapers" & 251 & settings and utils $(37 \%)$, wallpapers $(37 \%)$, personalize \\
\hline
\end{tabular}

elements' silhouette for each solution using $K$ as the number of clusters, and it selects the solution whose silhouette is closest to 1 .

\subsection{Resulting App Clusters}

Table 3 shows the list of clusters that CHABADA identifies for the 22,521 apps that we analyzed. Each of these 32 clusters contains apps whose descriptions contain similar topics, listed under "Most Important Topics". The percentages reported in the last column represent the weight of specific topics within each cluster.

The clusters we identified are quite different from the categories one would find in an app store such as the Google Play Store. Cluster 22 ("advertisements"), for instance, 
is filled with applications that do nothing but display ads in one way or another; these apps typically promise or provide some user benefit in return. Cluster 16 ("connection") represents all application that deal with Bluetooth, Wi-Fi, etc.; there is no such category in the Google Play Store. The several "wallpaper" clusters, from adult themes to religion, simply represent the fact that several apps offer very little functionality.

The Official New York Jets app ended up in Cluster 19, together with other applications that are mostly about sports. Table 3 lists the clusters of apps related by their descriptions in which we now can search for outliers with respect to their behavior.

\section{Identifying Anomalies by APIs}

After clustering apps based on similarity of their description topics, CHABADA searches for outliers regarding their actual behavior. Section 3.1 shows how CHABADA extracts API features from Android binaries. Section 3.2 describes how it filters APIs to be used as features, and how it weighs APIs according to their importance. Section 3.3 describes how CHABADA detects API outliers, while Section 3.4 describes how CHABADA can be used as a classifier.

\subsection{Extracting API Usage}

As discussed in the introduction, CHABADA uses static API usage as a proxy for behavior. Going for API usage is straightforward: while Android bytecode can also be subject to advanced static analysis such as information flow analysis and standard obfuscation techniques that easily thwart any static analysis, API usage has to be explicitly declared; in Android binaries, as in most binaries on other platforms, static API usage is easy to extract. For each Android application, CHABADA extracts the (binary) APK file with apktoo 6 , and with a smali parser extracts all API invocations.

\subsection{Sensitive and Rare APIs}

Using all API calls would result in too much information to represent the behavior of an application. Therefore, we focus on a subset of APIs only, namely sensitive APIs that

\footnotetext{
6https://code.google.com/p/android-apktool
} 
Table 4: Filtered APIs used in Official New York Jets that make this app an outlier in its cluster. Each application is associated to an anomaly score, which is explained in Section 3

\begin{tabular}{lr} 
com.yinzcam.nfl.jets & Anomaly Score $\mathbf{1 0 9 2 0 . 1}$ \\
\hline Feature importance: & \\
android.location.LocationManager.addGpsStatusListener() & 2900.00 \\
android.net.wifi.WifiManager.pingSupplicant() & 2900.00 \\
android.net.wifi.WifiManager.setWifiEnabled() & 1452.51 \\
android.telephony.SmsManager.sendTextMessage() & 1162.01 \\
java.lang.Runtime.exec() & 970.02
\end{tabular}

are governed by an Android permission setting. These APIs access sensitive information (such as the user's picture library, the camera, or the microphone) or perform sensitive tasks (altering system settings, sending messages, etc.) When installing an app, the user must explicitly permit usage of these APIs. For this purpose, each Android app includes a manifest file which lists the permissions that the application requires for its execution. To obtain the set of sensitive APIs, we relied on the work of Felt et al., who identified and used the mapping between permissions and Android methods [7]; CHABADA only considers a sensitive API to be used by the app if and only if it is declared in the binary and if its corresponding permission is requested in the manifest file. This allows to eliminate API calls that are used within third party libraries, and not used by the application directly.

As an example for such sensitive APIs, consider Table 4 These are some of the APIs used by the Official New York Jets app that are governed by a specific permission; through these APIs, the app accesses the GPS status, accesses the WiFi status, sends text messages, and executes arbitrary shell commands. The score of each API method expresses its impact on the overall anomaly score of the app. The anomaly score will be introduced and explained in the next section. Table 4 reports the top APIs ordered by anomaly score.

As each permission governs several APIs, going for permissions alone would be too few features to learn from; going for sensitive APIs allows a much more fine-grained characterization of the application behavior. Section 4 will provide empirical evidence that using APIs as features yields better results than using permissions. 
Filtering the APIs by considering only the sensitive ones, considerably limits the number of features. In [1] we show that it is possible to identify anomalous apps by using all sensitive APIs as features. We noticed, though, that among the sensitive APIs there exist some that are not as interesting as others. For instance, Internet access is governed by the "INTERNET" permission, and consequently any API requiring this permission should be considered as a sensitive one. However, most apps access Internet, and consequently accessing Internet is not an important feature for an application, since it has no discriminating power. On the contrary, sending text messages (governed by the "SEND-SMS" permission) is not a common feature among Android apps, and should thus be considered more than others. The cluster context, however, must be taken into account, since wallpaper applications, for instance, do not frequently use Internet connection, and consequently this could be a discriminating feature.

Removing common features would be too aggressive. Instead, we employ the idea of feature ranking based on statistical analysis. We then use different weights either to emphasize or diminish a particular feature. Our strategy is similar to IDF (inverse document frequency) [8], which is a part of the TF-IDF measure, well known in Information Retrieval. It is intended to reflect how important a word is to a document in a collection of documents (typically referred to as corpus).

Accordingly, we define weights with the following formula:

$$
\mathrm{W}_{a}=\log \frac{N}{\mathrm{df}_{a}}
$$

The weight $W_{a}$ of an API $a$ is obtained by dividing the total number of applications $(N)$ in a cluster by the number of applications $\operatorname{df}_{a}$ calling the API $a$, and then taking the logarithm of that quotient. Thus, the weight of a rare API is high, whereas the weight of a common API is likely to be low.

Section 4 provides empirical evaluation that using IDF helps CHABADA identifying the important features for anomaly detection.

\subsection{Distance-based Outlier Detection}

Now that we have all API features for all apps, the next step is to identify outliers that is, those applications whose API usage would be abnormal within their respective 
topic cluster. Since we have no notion about an underlying generating model for our data, it is reasonable to utilize a non-parametric approach to identify these outliers. Namely, we changed CHABADA such that it uses a distance-based technique [9], that is it uses the distance of an app to the other apps within the same cluster as a measure of anomaly. Distance-based approaches can identify outliers according to different definitions:

1. Outliers are those elements with fewer than $k$ neighbors, where a neighbor is an element that is within a maximum specified distance [9].

2. Outliers are those elements whose distance to their respective $k$-th nearest neighbor is highest. This is what is usually referred as the k-Nearest Neighbors $(k-N N)$ algorithm [10].

3. Outliers are those elements whose average distance to their respective $k$ nearest neighbors is highest [11].

CHABADA uses Orca, an open source framework that implements distance-based outlier detection [12]. The distance function of Orca computes the Euclidean distance of two apps in the feature space (i.e., the APIs). The first definition of outliers requires to specify the maximum neighborhood, and does not provide ranking scores for the outliers. The second definition does not take into account the local density of samples. Thus, CHABADA identifies anomalies considering the average distance of an element, i.e. an app in the cluster, to the $k$ nearest neighbors. The intuition is that if there are other samples that are close to the candidate in the feature space, then the sample is probably not an outlier. We use 5 as the value for $k$, as this number offers a good tradeoff between two extremes: a small value for $k$ would be too sensitive to noise, and would therefore miss many outliers; on the other hand, a high value for $k$ would regard almost any app as an outlier.

CHABADA uses the average distance to the 5 closest neighbors as the "anomaly" score of each app in the cluster, and it ranks the apps according to this score. The higher the score, the more anomalous the behavior of an app is. Some apps may be flagged as anomalies because they use few APIs that are never (or seldomly) used in the cluster. 
Others may be considered anomalies because they use combinations of APIs that do not occur frequently. Table 4 shows the anomaly score for the Official New York Jets app, and shows the features with the highest values, i.e., the APIs that have the highest impact on the final anomaly score.

By virtue of this distance-based technique, CHABADA can assign an anomaly score to each app within each cluster. These anomalies, though, are meant to be manually evaluated, and it is therefore critical to select a cutoff value. Apps whose anomaly score is above this cutoff value would be reported as anomalies, and the ones below this value would be considered normal. It is not trivial to select a cutoff value, as it strongly depends on the data. An easy solution would be to report as outliers a certain fraction of the data, namely the apps with the highest score. An alternative common approach would be to use quartile statistics.

The potential outliers here would be those apps whose score exceeds the third quartile by more than 1.5 times the interquartile range (third quartile minus first quartile). This is one of the standard measures for identifying outliers in a data set.

Within various clusters anomaly scores differ widely in their their range, contrast, as well as in their meaning, and unfortunately this makes it difficult to interpret and compare the results. In many cases, an identical outlier score in two different clusters can denote substantially different degrees of outlierness, depending on different local data distributions. Simple normalization for $k-N N$ does not result in good contrast between outliers and inlier scores, as it would just scale data onto the range $[0,1]$. A solution to this problem is to represent values as a probability of an application of being an outlier.

Following the approach proposed by Kriegel et al. [13] CHABADA transforms the anomaly scores into probabilities using Gaussian Scaling.

Without drawing any assumptions on the distribution of the data, we can suppose, according to the central limit theorem, that the computed anomaly scores have normal distribution. Given the sample mean $\mu$ and the sample standard deviation $\sigma$ of the set of anomaly scores $S$, we can use its cumulative distribution function and the "Gaussian error function" $\operatorname{erf}()$ to turn the anomaly score into a probability value:

$$
\mathrm{P}(s)=\max \left\{0, \operatorname{erf}\left(\frac{s-\mu}{\sqrt{2} \cdot \sigma}\right)\right\}
$$


All applications with non-zero probability are reported by CHABADA as outliers.

\subsection{CHABADA as a Malware Detector}

CHABADA can also be used to detect malware. For this task we use One-Class Support Vector Machine learning (OC-SVM) [14], which is an unsupervised machine learning technique to learn the features of one class of elements. The learned model can then be used to evaluate new apps, and the classifier would decide whether they are similar or different to the training set. Note how this is in contrast to the more common usage of Support Vector Machines as classifiers, where each app additionally has to be labeled as belonging to a specific class — say, "benign" vs. "malicious"—during training.

OC-SVMs have been successfully applied in various contexts that span from document classification [15] to automatic detection of anomalous Windows registry accesses [16]. In our context, the interesting feature of OC-SVM is that one can provide only samples of one class (say, of regular benign applications), and the classifier will be able to identify samples belonging to the same class, tagging the others as malware. OC-SVMs, therefore, are mainly used in those cases in which there exist many samples of one class of elements (e.g. benign applications), and not many samples of other classes (e.g. malicious applications).

The OC-SVM algorithm first projects input data into a high dimensional feature space via an appropriate kernel function, and considers the origin as the only sample different from the training data. It then identifies the maximal margin on the hyperplane that best separates the training data from the origin. The kernel mapping is necessary since usually data in the initial feature space are not separable via linear hyperplane division.

A training dataset containing anomalies would not result in a good model, since outliers could significantly influence the decision boundary of a OC-SVM. With the filtered APIs as features (as described in Section 3.2), CHABADA first identifies outliers, as described in Section 3.3, and then trains a OC-SVM within each cluster with the subset of the applications that were not tagged as outliers. Thus, the obtained model represents the APIs that are commonly used by the applications in that cluster. The 
resulting cluster-specific models are then used to classify new apps, that can be benign, and thus expected to have features that are similar to the trained model, or malicious, and thus expected to differ. Our OC-SVM uses a radial-bases-function (Gaussian) kernel, which is the most commonly used for OC-SVM. It has been shown that for this type of classification it performs better than other kernels (e.g., linear or polynomial) [17], and this was also confirmed by our experiments.

The Gaussian kernel size, which represents how tight the boundary should fit over the training data, has to be manually specified. It is essential to properly select this parameter, as it highly influences the performance of the classifier. Too small values would lead to overfitting, while a larger value of the kernel size would give a smoother decision surface and more regular decision boundary.

Selecting the kernel size of OC-SVM remains an open problem. In CHABADA we used the default kernel size value, as proposed by Schölkopf [18], which is equal to the inverse of the feature space dimension. Using the default kernel size, however, does not take into account the arrangement of data, and it is therefore not optimal. According to Caputo et al. [19], the optimal value of the inverse kernel size $\gamma$ lies between the 0.1 and 0.9 quantile of the statistics involving distance from training samples to their center point. We thus now set $\gamma$ to the mean of quantiles of this range, and this improves significantly the results, as we will show in the evaluation.

Section 4 will show how $\gamma$ influences the classification ability of CHABADA.

\section{Evaluation}

To evaluate the effectiveness of CHABADA, we investigated the following main research questions:

RQ1 Can CHABADA effectively identify anomalies (i.e., mismatches between description and behavior) in Android applications? For this purpose, we visualized how often anomalies occur in each cluster, and we manually analyzed the top anomalies Section 4.1. 
RQ2 Are sensitive and rare APIs the appropriate features to detect anomalies? We compare the results obtained with the sensitive and rare set of APIs, as described in Section 3.2, against different sets of features (Section 4.2).

RQ3 Can CHABADA be used to identify malicious Android applications, and how does the improved technique compare to [1]? For this purpose, we included in our test set of applications a set of known malware, and we ran OC-SVM as a classifier. We show how the improvements proposed in this paper lead to more accurate results Section 4.3.

\subsection{RQ1: Anomaly Detection}

To identify anomalies (i.e., mismatches between description and behavior) in Android applications, we ran CHABADA on all 32 clusters, as described in Section 3

The best way to evaluate whether the outliers that CHABADA identifies are indeed anomalous applications, is via manual evaluation. We did this in our conference paper [1], and we found similar results using the new outlier detection mechanism described in Section 3 Outliers fall into the following categories: 1) spyware applications, which mainly collect sensitive information about the user by means of third party advertisement libraries 2) very unusual applications, i.e., applications that, although benign, behave differently from other similar applications 3) benign applications that were assigned to the wrong topic cluster, and thus behave differently from other applications in the cluster. The third category shows that we should improve the first step of the CHABADA approach (Section 2), but the other two categories show that the outliers reported by CHABADA indeed have suspicious behavior, and should therefore be further analyzed. We now list few examples of applications that appeared as top outliers in the corresponding clusters. We list the cluster number, as reported in Table 3 , the package identified of the application, the anomaly score, a brief description (which is a one line summary of the original one), the list of the top anomalous features, and a brief comment: 


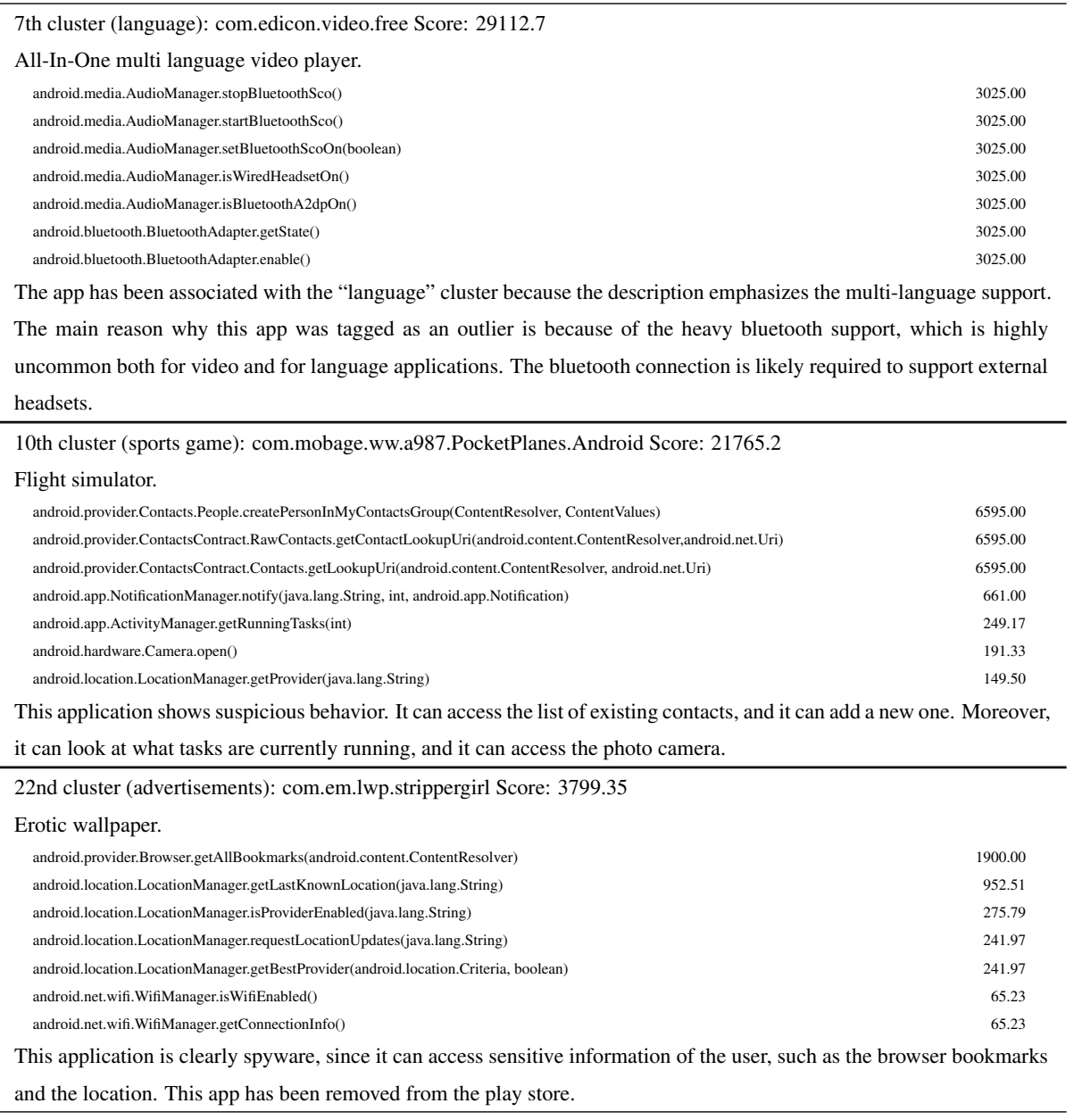

Beside a qualitative analysis of the results, we tried to estimate the ability of CHABADA to detect anomalies by plotting the anomaly scores of the outliers of each cluster. Intuitively, a good result would be to have few outliers with high anomaly scores within each cluster. A worse result, instead, would be to have lots of outliers whose anomaly score is not too high. In the second case, in fact, it would mean that there is no clear border between the outliers and the normal applications. Figure 3 shows such plot. We report the anomaly scores (on the y axis) of all the apps grouped per cluster (on the $\mathrm{x}$ axis) scaled such that all clusters have the same width, in order to ease the comparison.

The cluster ids correspond to the ones listed in Table 3 . 


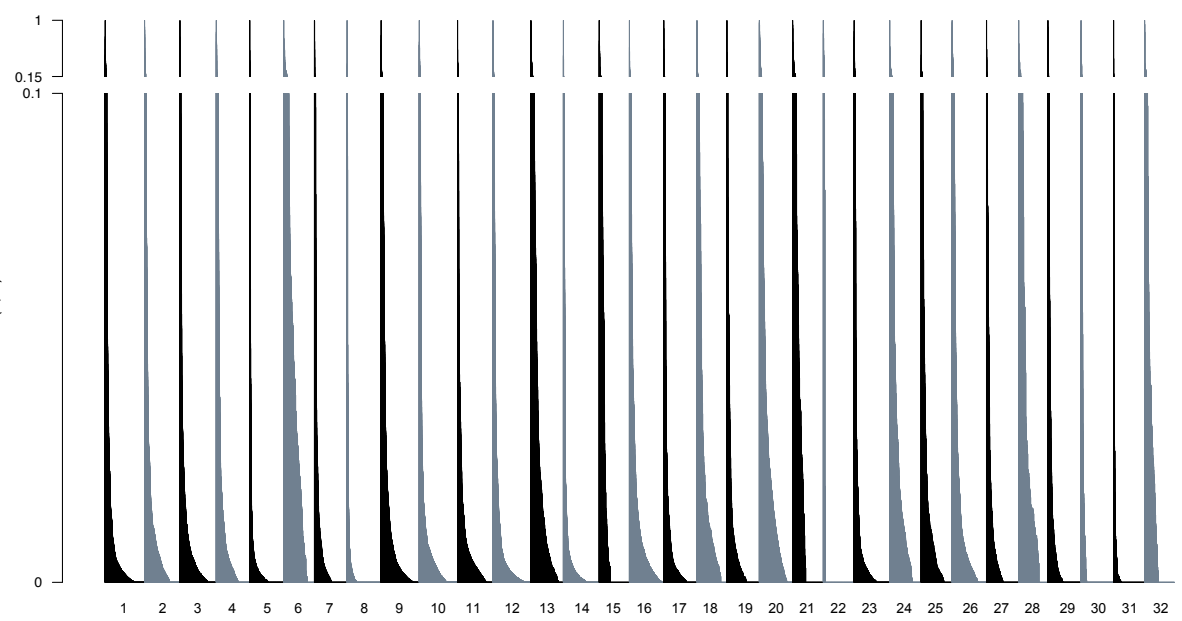

Figure 3: Apps grouped per cluster (x axis) according to their anomaly score (y axis).

As expected, the results vary depending on the cluster. There are clusters in which the outliers are clear anomalies, as for instance clusters 5 or 29, and others for which there are lots of outliers with high anomaly scores, for instance clusters 6 and 20. When clusters have too many outliers, they lack a proper model of "normal" behavior, and as a consequence our technique might be less effective in in such clusters.

\subsection{RQ2: Feature Selection}

Section 3.2 describes how we select the list of features to detect anomalous applications within a cluster. In [1] we considered the sensitive API usage as binary features (i.e., 1 if the app used the API at least once, 0 otherwise), while we now use IDF to weigh APIs. To evaluate whether the feature selection is reasonable, we ran the anomaly detection process on each cluster with three different sets of features:

1. We considered binary values for sensitive API usage. These are the features used in our conference paper [1] We refer to this setting as api-binary.

2. We weighed sensitive API according to IDF. This is, in our opinion, the optimal set. We refer to this setting as api-idf. 

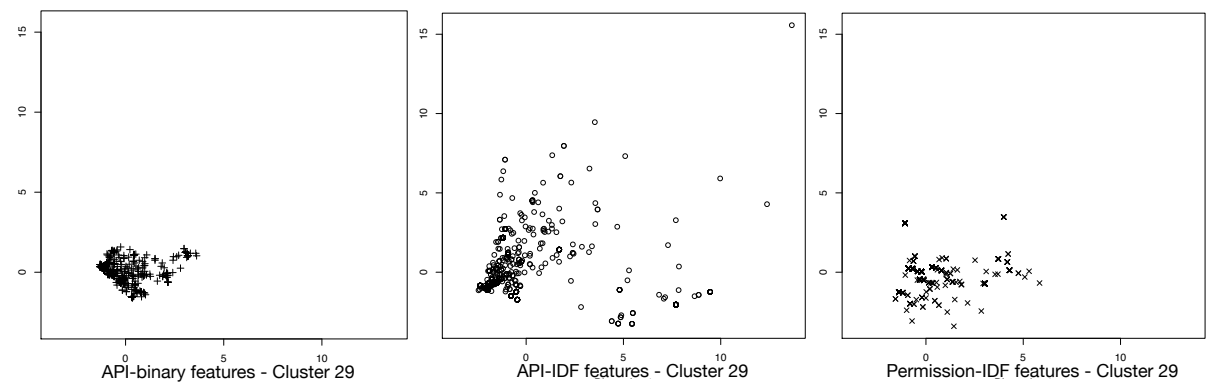

Figure 4: Plots of the distances among apps in clusters 29. From left to right, these plots show the distance between apps when using api-binary features, as in [1], api-idf, as described in Section 3.2 and permission-idf.

3. We used permissions instead of APIs, and we weighed permissions with IDF. With this, we wanted to evaluate whether using permissions could be considered a valid alternative to using APIs. We refer to this setting as permission-idf.

Comparing different settings is not trivial, as it would require an extensive manual inspection. We instead visually compare the distance-based plots of several clusters. Figure 4 shows the plots of cluster 29 , which is one of the clusters for which we have better results. From left to right, the plots show the three different settings described above: api-binary, api-idf, and permission-idf.

We used multi-dimensional scaling, which is a statistical technique used to visualize dissimilarity of multi-dimensional data. This allowed us to plot data in two dimensions, and at the same time preserve the original distances in the multi-dimensional space as accurately as possible [20].

As we can see, by using permissions or APIs with IDF it is possible to differentiate the anomalies better, since the distance between the outliers and the rest of the cluster is emphasized. Among the two options, though, it is better to use APIs instead of permissions. In the next Section we provide more evidence that using IDF can lead to better results.

\subsection{RQ3: Malware Detection}

Let us now turn to RQ3: Can CHABADA be used to identify malicious Android applications? And do the improvements proposed in this paper lead to better results 
that the ones presented in [1]? For this purpose, we used the dataset of Zhou et al. [21] containing more than 1,200 known malicious apps for Android. This is the same dataset that we used in the original CHABADA paper [1]. In their raw form, these apps lack metadata such as title or description. Since many of these apps are repackaged versions of original apps, we were able to collect the appropriate description from the Google Play Store. We used the title of the application and the package identifier to search for the right match in the Store. For 72 cases we could find exactly the same package identifier, and for 116 applications we found applications whose package identifiers were very similar. We manually checked that the match was correct. As with our original set of "benign" apps Section 2.1, we only kept those applications with an English description in the set, reducing it to 172 apps.

As a malware detector, we used the OC-SVM classifier, as described in Section 3.4 . Within each cluster, we trained the model using only the applications that were not tagged as outliers by the distance-based algorithm. Following K-fold validation, we partitioned the entire set of non outlier benign applications in 10 subsets, and we used 9 subsets for training the model and 1 for testing. We then included the malicious applications in the test set, and we ran this 10 times, each time considering a different subset for testing. What we thus simulated is a situation in which the malware attack is entirely novel, and CHABADA must correctly identify the malware as such without knowing previous malware patterns. The number of malicious applications are not be equally distributed across clusters, as malicious applications are assigned to clusters depending on their descriptions. In our evaluation setting, with our data set, the number of malicious applications per cluster spans from 0 to 39.

To evaluate the performance of a classifier we use the standard approach of the Receiver Operating Characteristic (ROC) [22]. A ROC curve depicts the relative tradeoffs between benefits (True Positives) and costs (False Positives). Figure 5 shows the results of our experiments in the form of this ROC curve, which plots the true positives rate against the false positive rate considering different thresholds.

Figure 5 shows the ROC curves of the worst and best clusters (cluster 16 and 7 respectively), and the average performance on all clusters. To obtain these numbers, we computed the average over 10 different runs. We also report the Area Under the 


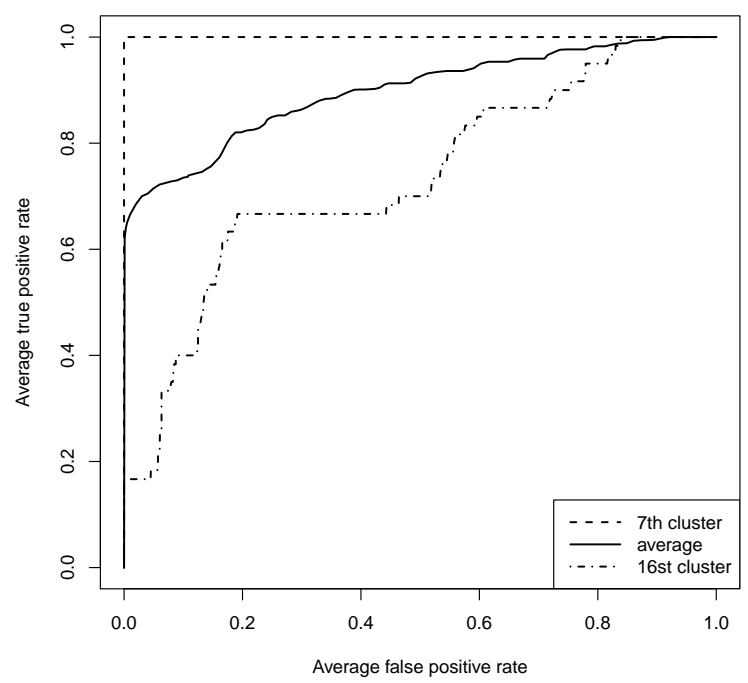

Figure 5: ROC curves, representing the fraction of true positives out of the total actual positives vs. the fraction of false positives out of the total actual negatives. We report the average performance of the classifier across all clusters, for which the Area Under Curve (AUC) is equal to 0.87 . We also plot the performance for the worst (16st cluster) and best clusters (7th cluster).

ROC curve (AUC) [22] metric, which can expose the predictive accuracy obtained by the classifier. When AUC is equal to 1.0, it means that the classification was perfect, while an area of 0.5 represents a worthless test. CHABADA as a classifier has a very good performance, as the AUC is 0.87 on the considered dataset, and thus we can claim that it is effective at detecting malware, reporting only limited false positives.

In our first paper, CHABADA used the set of sensitive Android APIs as binary features. Moreover, we did not filter anomalous applications when training the model for classification, and we used the default (and therefore not optimal) values for the kernel size and marginal error for OC-SVM. Section 3 described all the improvements that we implemented in the new release of CHABADA. To evaluate the effectiveness of our technique as a malware detector, we evaluate how such improvements had an impact on the final result. 


\begin{tabular}{|c|c|c|c|c|c|c|c|c|}
\hline \multirow[t]{2}{*}{ Filter } & \multirow[t]{2}{*}{$\gamma$} & \multirow[t]{2}{*}{$\nu$} & \multicolumn{2}{|c|}{$\begin{array}{l}\text { True Positive Rate } \\
\text { (Malware } \\
\text { recognized as such) }\end{array}$} & \multicolumn{2}{|c|}{$\begin{array}{l}\text { True Negative Rate } \\
\text { (Benignware } \\
\text { recognized as such) }\end{array}$} & \multicolumn{2}{|c|}{$\begin{array}{l}\text { Geometric } \\
\text { accuracy }\end{array}$} \\
\hline & & & IDF & Binary & IDF & Binary & IDF & Binary \\
\hline - & default & 0.15 & 0.610 & 0.564 & 0.845 & 0.841 & 0.718 & 0.689 \\
\hline- & optimal & 0.05 & 0.592 & 0.484 & 0.889 & 0.915 & 0.726 & 0.666 \\
\hline- & optimal & 0.1 & 0.609 & 0.539 & 0.870 & 0.869 & 0.728 & 0.684 \\
\hline+ & default & 0.02 & 0.633 & 0.535 & 0.962 & 0.96 & 0.780 & 0.716 \\
\hline+ & default & 0.1 & 0.726 & 0.637 & 0.890 & 0.885 & 0.804 & 0.750 \\
\hline+ & optimal & 0.02 & 0.737 & 0.695 & 0.890 & 0.902 & 0.810 & 0.791 \\
\hline+ & optimal & 0.05 & 0.738 & 0.752 & 0.884 & 0.887 & 0.808 & 0.791 \\
\hline+ & optimal & 0.1 & 0.752 & 0.738 & 0.850 & 0.856 & 0.799 & 0.795 \\
\hline+ & optimal & 0.15 & 0.810 & 0.771 & 0.814 & 0.813 & 0.812 & 0.792 \\
\hline
\end{tabular}

Table 5 shows the detailed results of the evaluation considering different parameters. The first column (Filter) lists whether malware detection ran on filtered data. The + sign means that we ran the anomaly detection first, and we removed the outliers from the training set. The - sign means that we considered all the applications, as we did in [1]. The second column lists whether the $\gamma$ parameter of OC-SVM was automatically selected to have optimal results, or whether the default value was selected, as in [1]. $\gamma$ is related to the kernel size, as explained in Section 3.3. The third column lists the value assigned to the $\nu$ parameter, which can be specified in OC-SVM. The parameter $\nu$ is an upper bound on the fraction of margin errors in training data and a lower bound of the fraction of support vectors relative to the total number of training examples. Assigning a small value to $\nu$ will produce less false positives and likely more false negatives, while assigning greater values to $\nu$ would have the opposite effects. In [1] we used the default value. The last six columns report the results obtained with the corresponding settings using APIs as binary features (as in [1]) or weighing them using IDF (as explained in Section 3.2). We report the True Positive Rate (TPR) (i.e. the fraction of malicious Android apps that were recognized as such), the True Negative Rate (TNR) (i.e. the fraction of benign Android apps that were recognized as such), and the Geometric Accuracy. We report the geometric accuracy because we have highly unbalanced datasets (malicious vs. benign applications), and therefore common 
accuracy metrics would have distorting effects [23]. The geometric accuracy can be computed with the following formula:

$$
g=\sqrt{T P R \times T N R}
$$

Just as we did for the plot in Figure 5, we report the average values of 10 runs. The first highlighted row reports the results and the setting used in the original CHABADA paper. This, in essence, represents the baseline for our malware detector. This row also reports how only applying IDF to the used features would have changed the results. As highlighted in bold, without any of the improvements that we described in this paper we could detect $56.4 \%$ of malware as such, and $84.1 \%$ of benignware as such.

The other two highlighted rows in Table 5 show the results of the using the improvements described in this paper. In essence, we propose two possible settings, which both achieve an accuracy of over 79\% (instead of $69 \%$ as in [1]). Using a small $\nu$ value would report a limited number of false positives $(\mathbf{1 1 \%})$, and at the same time would detect a high number of malicious apps (73.7\%). Using a bigger $\nu$ value, instead, would increase the number of detected malicious apps up to $\mathbf{8 1 \%}$, but at the cost of reporting more false positives (19\%).

With the improvements proposed in this paper, CHABADA correctly recognizes $73.7 \%$ of malware as such, with only $11 \%$ of false positives.

The results in Table 5 clearly highlight the following facts:

- Training the OC-SVM model without the identified outliers clearly improves the classifier results. This is obvious, as removing outliers helps creating a model that better represents the core features of the cluster.

- Tuning the $\gamma$ value, which relates to the kernel size of the OC-SVM model, can also lead to significantly better results.

- Assigning weights to APIs with IDF produces consistently better results than using APIs as binary features.

Thus, the three main improvements that we propose in this paper (filtering outliers, optimal selection of the OC-SVM kernel size, and using IDF to assign weights to APIs) can produce results that are significantly better than the ones we presented in [1]. 
Choosing parameter $\nu$ is a matter of taste, as it depends whether it is more important to have as little false positives as possible (and consequently choose a smaller value for $\nu$ ) or to have as little false negatives as possible (and consequently choose a higher value for $\nu$ ). In this context it is probably better to detect as many malicious applications as possible, and consequently a higher value of $\nu$ should be chosen (last highlighted row in Table 57. However, if it is desirable to lower the number of false positives, a lower value of $\nu$ can be selected, and still have comparable effectiveness (second highlighted row in Table 5). This tradeoff can be tuned using the ROC curve of Figure 5 .

\subsection{Limitations and Threats to Validity}

We inherit most of the limitations of the original CHABADA paper, and we include a new one that comes with the filtering phase. The most important threats and limitations are listed below.

Grey area in classification. Filtering outliers when building the model to classify new applications leads to significant improvements in malware detection. The consequence of this gain, however, is that the outlier apps that are filtered out in first place cannot be classified. More precisely, these apps are reported in a "grey area", that is, they are suspicious since they are not as the majority of the apps in the same cluster, but CHABADA does not report them as clearly malicious.

External validity. CHABADA relies on establishing a relationship between description topics and program features from existing, assumed mostly benign, applications. We cannot claim that said relationships could be applied in other app ecosystems, or be transferable to these. We have documented our steps to allow easy replication of our approach.

Free apps only. Our sample of 22,521 apps is based on free applications only; i.e. applications that need to generate income through ads, purchases, or donations. Not considering paid applications makes our dataset biased. However, the bias would shift "normality" more towards apps supported by ads and other income methods, which are closer to undesired behavior exposed by malware. Our results 
thus are conservative and would rather be improved through a greater fraction of paid applications, which can be expected to be benign.

App and malware bias. Our sample also only reflects the top 150 downloads from each category in the Google Play Store. This sample is biased towards frequently used applications, and towards lesser used categories; likewise, our selection of malware (Section 4) may or may not be representative for current threats. Not knowing which actual apps are being used, and how, by Android users, these samples may be biased. Again, we allow for easy reproduction of our approach.

Native code and obfuscation. We limit our analyses to the Dalvik bytecode. We do not analyze native code. Hence, an application might rely on native code or use obfuscation to perform covert behavior; but then, such features may again characterize outliers; also, neither of these would change the set of APIs that must be called.

Static analysis. As we rely on static API usage, we suffer from limitations that are typical for static analysis. In particular, we may miss behavior induced through reflection, i.e. code generated at runtime. Although there exist techniques to statically analyze Java code using reflection, such techniques are not directly applicable with Android apps [24]; in the long run, dynamic analysis paired with test generation may be a better option.

Static API declarations. Since we extract API calls statically, we may consider API calls that are never executed by the app. Checking statically whether an API is reached is an instance of the (undecidable) halting problem. As a workaround, we decided to consider an API only if the corresponding permission is also declared in the manifest.

Sensitive APIs. Our detection of sensitive APIs Section 3.2 relies on the mapping by Felt et al. [7], which now, two years later, may be partially outdated. Incorrect or missing entries in the mapping would make CHABADA miss or misclassify relevant behavior of the app. 


\section{Related Work}

While this work may be the first to generally check app descriptions against app behavior, it builds on a history of previous work combining natural language processing and software development.

\subsection{Mining App Descriptions}

Most related to our work is the AsDroid prototype, which can detect malicious behavior by identifying mismatches between the program behavior and the user interface [25]. CHABADA and AsDroid share the same final intent, but AsDroid checks the text in GUI components, while we use the program description. AsDroid, though, focuses on few permissions, and works only if the GUI elements contain textual keywords. CHABADA, instead, uses the application description, which is always available, and works with any permission or API call.

Also very related to our work is the WHYPER framework of Pandita et al. [26]. Just like our approach, WHYPER attempts to automate the risk assessment of Android apps, and applies natural language processing to app descriptions. The aim of WHYPER is to tell whether the need for sensitive permissions (such as accesses to contacts or calendar) is motivated in the application description. In contrast to CHABADA, which fully automatically learns which topics are associated with which APIs (and by extension, which permissions), WHYPER requires manual annotation of sentences describing the need for permissions. Also, CHABADA goes beyond permissions in two ways: first, it focuses on APIs, which provide a more detailed view, and it aims for general mismatches between expectations and implementations.

The very idea of app store mining was introduced one year earlier when Harman et al. mined the Blackberry app store [27]. They focused on app meta-data to find patterns such as a correlation consumer rating and the rank of app downloads, but would not download or analyze the apps themselves.

Our characterization of "normal" behavior comes from mining related applications; in general, we assume what most applications in a well-maintained store do is also what most users would expect to be legitimate. In contrast, recent work by Lin et al. [28] 
suggests crowdsourcing to infer what users expect from specific privacy settings; just like we found, Lin et al. also highlight that privacy expectations vary between app categories. Such information from users can well complement what we infer from app descriptions.

\subsection{Behavior/Description Mismatches}

Our approach is also related to techniques that apply natural language processing to infer specifications from comments and documentation. Lin Tan et al. [29] extract implicit program rules from program corpora and use these rules to automatically detect inconsistencies between comments and source code, indicating either bugs or bad comments. Rules apply to ordering and nesting of calls and resource accesses (" $f_{a}$ must not be called from $f_{b}$ ").

Høst and Østvold [30] learn from program corpora which verbs and phrases would normally be associated with specific method calls, and used these to identify misnamed methods.

Pandita et al. [31] identify sentences that describe code contracts from more than 2,500 sentences of API documents; these contracts can be checked either through tests or static analysis.

All these approaches compare program code against formal program documentation, whose semi-formal nature makes it easier to extract requirements. In contrast, CHABADA works on end-user documentation, which is decoupled from the program structure.

\subsection{Detecting Malicious Apps}

There is a large body of industrial products and research prototypes that focus on identifying known malicious behavior. Most influential for our work was the paper by Zhou and Jiang [21], who use the permissions requested by applications as a filter to identify potentially malicious applications; the actual detection uses static analysis to compare sequences of API calls against those of known malware. In contrast to all these approaches, CHABADA identifies outliers even without knowing what makes malicious behavior. 
The TAINTDROID system [32] tracks dynamic information flow within Android apps and thus can detect usages of sensitive information. Using such dynamic flow information would yield far more precise behavior insights than static API usage; similarly, profilers such as ProfileDroid [33] would provide better information; however, both TAINTDROID and ProfileDroid require a representative set of executions. Integrating such techniques in CHABADA, combined with automated test generation [34, 35, 36, 37], would allow to learn normal and abnormal patterns of information flow; this is part of our future work Section 6 .

\section{Conclusion and Future Work}

By clustering apps according to description topics, and identifying outliers by API usage within each cluster, our CHABADA approach effectively identifies applications whose behavior would be unexpected given their description. In [1] we have identified several examples of false and misleading advertising; and as a side effect, obtained a novel effective detector for yet unknown malware. This paper presented several improvements on the original technique, and thus allows to have a more powerful malware detector.

In future we plan to provide better techniques to cluster applications according to their descriptions. This should improve the ability of CHABADA to identify relevant abnormal behaviors. Furthermore, we plan to integrate dynamic information in the approach, thus overcoming the known limitations of static analysis.

The dataset that we used for our evaluation, as well as a list of more detailed results are available on the CHABADA web site:

http://www.st.cs.uni-saarland.de/appmining/chabada/

\section{Acknowledgments.}

This work was funded by the European Research Council (ERC) Advanced Grant "SPECMATE - Specification Mining and Testing". 


\section{References}

[1] A. Gorla, I. Tavecchia, F. Gross, A. Zeller, Checking app behavior against app descriptions, in: ACM/IEEE International Conference on Software Engineering (ICSE), 2014, pp. 1025-1035. doi:10.1145/2568225.2568276.

[2] C. Manning, P. Raghavan, H. Schütze, Introduction to Information Retrieval, Cambridge University Press, 2008.

[3] D. M. Blei, A. Y. Ng, M. I. Jordan, Latent Dirichlet allocation, Journal of Machine Learning Research 3 (2003) 993-1022.

[4] A. K. McCallum, Mallet: A machine learning for language toolkit, http://mallet.cs.umass.edu (2002).

[5] J. B. MacQueen, Some methods for classification and analysis of multivariate observations, in: L. M. L. Cam, J. Neyman (Eds.), Berkeley Symposium on Mathematical Statistics and Probability, Vol. 1, University of California Press, 1967, pp. 281-297.

[6] P. Rousseeuw, Silhouettes: a graphical aid to the interpretation and validation of cluster analysis, Journal of Computational and Applied Mathematics 20 (1) (1987) 53-65. doi:10.1016/0377-0427(87)90125-7

[7] A. P. Felt, E. Chin, S. Hanna, D. Song, D. Wagner, Android permissions demystified, in: ACM Conference on Computer and Communications Security (CCS), ACM, New York, NY, USA, 2011, pp. 627-638. doi:10.1145/2046707. 2046779

[8] G. Salton, M. McGill, Introduction to Modern Information Retrieval, McGraw-Hill Book Company, 1983.

[9] E. M. Knorr, R. T. Ng, Algorithms for mining distance-based outliers in large datasets, in: Proceedings of the 24rd International Conference on Very Large Data Bases (VLDB), Morgan Kaufmann Publishers Inc., 1998, pp. 392-403. 
[10] S. Ramaswamy, R. Rastogi, K. Shim, Efficient algorithms for mining outliers from large data sets, in: Proceedings of the 2000 ACM SIGMOD International Conference on Management of Data (SIGMOD), ACM, 2000, pp. 427-438. doi: $10.1145 / 342009.335437$

[11] F. Angiulli, C. Pizzuti, Fast outlier detection in high dimensional spaces, in: Proceedings of the 6th European Conference on Principles of Data Mining and Knowledge Discovery (PKDD), Springer-Verlag, 2002, pp. 15-26.

[12] S. D. Bay, M. Schwabacher, Mining distance-based outliers in near linear time with randomization and a simple pruning rule, in: 9th ACM SIGKDD International Conference on Knowledge Discovery and Data Mining (KDD), ACM, 2003, pp. 29-38. doi:10.1145/956750.956758.

[13] E. S. Hans-Peter Kriegel, Peer Kröger, A. Zimek, Interpreting and unifying outlier scores, in: 11th SIAM International Conference on Data Mining (SDM), 2011, SIAM, 2011, pp. 13-24. doi:10.1137/1.9781611972818

[14] B. Schölkopf, J. C. Platt, J. C. Shawe-Taylor, A. J. Smola, R. C. Williamson, Estimating the support of a high-dimensional distribution, Neural Computation 13 (7) (2001) 1443-1471. doi:10.1162/089976601750264965

[15] L. M. Manevitz, M. Yousef, One-class SVMs for document classification, Journal of Machine Learning Research 2 (2002) 139-154.

[16] K. A. Heller, K. M. Svore, A. D. Keromytis, S. J. Stolfo, One class support vector machines for detecting anomalous windows registry accesses, in: ICDM Workshop on Data Mining for Computer Security (DMSEC), 2003.

[17] D. Tax, P. Juszczak, Kernel whitening for one-class classification, in: S.-W. Lee, A. Verri (Eds.), Pattern Recognition with Support Vector Machines, Vol. 2388 of Lecture Notes in Computer Science, Springer Berlin Heidelberg, 2002, pp. 40-52. doi:10.1007/3-540-45665-1_4 
[18] B. Schölkopf, A. J. Smola, Learning with Kernels: Support Vector Machines, Regularization, Optimization, and Beyond, MIT Press, Cambridge, MA, USA, 2001.

[19] B. Caputo, K. Sim, F. Furesjo, A. Smola, Appearance-based object recognition using svms: Which kernel should i use?, in: NIPS workshop on Statistical methods for computational experiments in visual processing and computer vision, 2002.

[20] T. Cox, M. Cox, Multidimensional Scaling, Chapman \& Hall, 2001.

[21] Y. Zhou, X. Jiang, Dissecting Android malware: Characterization and evolution, in: IEEE Symposium on Security and Privacy (SP), IEEE Computer Society, Washington, DC, USA, 2012, pp. 95-109. doi:10.1109/SP.2012.16.

[22] T. Fawcett, An introduction to roc analysis, Pattern Recogn. Lett. 27 (8) (2006) 861-874. doi:10.1016/j.patrec.2005.10.010

[23] M. Kubat, S. Matwin, Addressing the curse of imbalanced training sets: onesided selection, in: In Proceedings of the Fourteenth International Conference on Machine Learning, Morgan Kaufmann, 1997, pp. 179-186.

[24] E. Bodden, A. Sewe, J. Sinschek, H. Oueslati, M. Mezini, Taming reflection: Aiding static analysis in the presence of reflection and custom class loaders, in: ACM/IEEE International Conference on Software Engineering (ICSE), ACM, 2011, pp. 241-250. doi:10.1145/1985793.1985827

[25] J. Huang, X. Zhang, L. Tan, P. Wang, B. Liang, Asdroid: Detecting stelthy behaviors in android applications by user interface and program behavior contradiction, in: ACM/IEEE International Conference on Software Engineering (ICSE), 2014, pp. 1036-1046. doi:10.1145/2568225.2568301.

[26] R. Pandita, X. Xiao, W. Yang, W. Enck, T. Xie, WHYPER: Towards automating risk assessment of mobile applications, in: USENIX Security Symposium, 2013, pp. 527-542. 
[27] M. Harman, Y. Jia, Y. Zhang, App store mining and analysis: MSR for app stores, in: IEEE Working Conference on Mining Software Repositories (MSR), 2012, pp. 108-111. doi:10.1109/MSR.2012.6224306.

[28] J. Lin, S. Amini, J. I. Hong, N. Sadeh, J. Lindqvist, J. Zhang, Expectation and purpose: understanding users' mental models of mobile app privacy through crowdsourcing, in: ACM Conference on Ubiquitous Computing (UbiComp), ACM, 2012, pp. 501-510. doi:10.1145/2370216.2370290.

[29] L. Tan, D. Yuan, G. Krishna, Y. Zhou, /* iComment: Bugs or bad comments? */, in: ACM SIGOPS Symposium on Operating Systems Principles (SOSP), 2007, pp. $145-158$.

[30] E. W. Høst, B. M. Østvold, Debugging method names, in: European Conference on Object-Oriented Programming (ECOOP), Springer, 2009, pp. 294-317.

[31] R. Pandita, X. Xiao, H. Zhong, T. Xie, S. Oney, A. Paradkar, Inferring method specifications from natural language API descriptions, in: ACM/IEEE International Conference on Software Engineering (ICSE), 2012.

[32] W. Enck, P. Gilbert, B.-G. Chun, L. P. Cox, J. Jung, P. McDaniel, A. N. Sheth, TaintDroid: an information-flow tracking system for realtime privacy monitoring on smartphones, in: USENIX conference on Operating Systems Design and Implementation (OSDI), USENIX Association, 2010, pp. 1-6.

[33] X. Wei, L. Gomez, I. Neamtiu, M. Faloutsos, ProfileDroid: multi-layer profiling of Android applications, in: ACM Annual International Conference on Mobile Computing and networking (MobiCom), ACM, New York, NY, USA, 2012, pp. 137-148. doi:10.1145/2348543.2348563.

[34] C. Hu, I. Neamtiu, Automating GUI testing for Android applications, in: International Workshop on Automation of Software Test (AST), ACM, New York, NY, USA, 2011, pp. 77-83. doi:10.1145/1982595.1982612.

[35] W. Yang, M. R. Prasad, T. Xie, A grey-box approach for automated GUI-model generation of mobile applications, in: International Conference on Fundamental 
Approaches to Software Engineering (FASE), Springer-Verlag, Berlin, Heidelberg, 2013, pp. 250-265. doi:10.1007/978-3-642-37057-1_19.

[36] A. Machiry, R. Tahiliani, M. Naik, Dynodroid: an input generation system for Android apps, in: European Software Engineering Conference held jointly with ACM SIGSOFT International Symposium on Foundations of Software Engineering (ESEC/FSE), ACM, New York, NY, USA, 2013, pp. 224-234. doi:http: //dx.doi.org/10.1145/2491411.2491450.

[37] D. Amalfitano, A. R. Fasolino, P. Tramontana, S. De Carmine, A. M. Memon, Using GUI ripping for automated testing of Android applications, in: IEEE/ACM International Conference on Automated Software Engineering (ASE), ACM, New York, NY, USA, 2012, pp. 258-261. doi:10.1145/2351676.2351717. 